\title{
Richtlinien für Autoren
}

\section{Verhaltenstherapie}

Verhaltenstherapie 2012;22

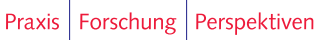

\section{Einreichung von Manuskripten}

VERHALTENSTHERAPIE veröffentlicht ausgewählte experimentelle, theoretische und anwendungsbezogene Arbeiten sowie Falldarstellungen zu allen Aspekten der Verhaltenstherapie und Verhaltensmedizin.

Manuskripte bitte online einreichen: www.karger.com/ver

Sollten Sie Probleme mit der Online-Einreichung haben, wenden Sie sich bitte an das Editorial Office:

S. Karger Verlag für Medizin und Naturwissenschaften $\mathrm{GmbH}$

Anna Piepiork

P.O. Box

79095 Freiburg (Deutschland)

Tel. +497614520726

Fax +497614520714

a.piepiorka@karger.com

Rubriken und Umfang

Die Manuskripte sollen in 11/2-zeiligem Abstand mit beidseitig je $3 \mathrm{~cm}$ Rand, Schrift 12 pt eingereicht werden.

Folgender Umfang sollte nicht überschritten werden:

\begin{tabular}{lll}
\hline & Druckseiten & Entspricht Zeichen* \\
\hline Original- und Übersichtsarbeiten & 8 & 48000 \\
Kasuistiken & 7 & 42000 \\
Für die Praxis & 7 & 42000 \\
Diskussionsforum & 5 & 30000 \\
Kurzberichte & 3 & 18000 \\
Fragebögen und Skalen & 2 & 12000 \\
\hline
\end{tabular}

*Einschließlich deutscher und englischer Zusammenfassung, Abbildungen, Tabellen und Literaturzitate. Pro Tabelle und Abbildung werden pauschal 1500 Zeichen gezählt.

Für Umfangsüberschreitungen wird dem Autor ein Mehrkostenanteil in Rechnung gestellt.

Kasuistiken sollen für die Praxis interessante Einzelfallanalysen darstellen und folgende Punkte enthalten

1. Beschreibung des Störungsbilds,

2. Differentialdiagnose,

3. hypothetisches Bedingungsmodell (Verhaltensanalyse),

4. Interventionsprinzipien,

5. Therapieergebnisse,

6. Schlussfolgerungen.

Innovative Behandlungsansätze sollen hervorgehoben werden. Notwendige emotionale Prozesse im Therapieverlauf sollen beschrieben werden. Angaben zu Therapieerfolgsmessungen zu verschiedenen Messzeitpunkten (prä/prozessorientiert/post) sind wünschenswert. Eine Literaturliste mit 5 Zitaten ist für Kasuistiken ausreichend.

Fragebögen und Skalen werden auf der «News and Features» der Zeitschrift zum freien Download veröffentlicht. Hintergrundinformationen dazu (Gütekriterien, Anwendung, Erfahrungen, etc.) erscheinen im gedruckten Heft und sollen 2 Druckseiten nicht überschreiten.

\section{Bedingungen}

Über die Annahme wissenschaftlicher Beiträge wird aufgrund mehrerer Gutachten entschieden (peer-review)

Der Verlag geht davon aus, dass eingereichte Manuskripte weder gleichzeitig bei einem anderen Journal begutachtet werden noch bereits ganz oder teilweise anderswo publiziert sind. Die Einreichung eines Beitrags impliziert, dass die Autoren alleinige Inhaber des Copyrights sind und dass der Artikel nicht in Rechte Dritter eingreift, inklusive des Copyrights und des Urheberrechtes.

Es ist Sache des Autors, die Nachdruckerlaubnis für Abbildungen, Tabellen usw. aus anderen Publikationen zu beschaffen.

Mit der Annahme des Manuskripts zur Publikation gehen alle Nutzungsrechte auf den Verlag über. Der Verlag hat das Recht, den Artikel in der vorliegenden oder in überarbeiteter Form zu reproduzieren und sich als Erstherausgeber zu bezeichnen. Die Nutzungsrechte sind weltweite Rechte und zeitlich unbegrenzt. Sie beziehen sich sowohl auf alle Print- als auch auf elektronische Offline- und Onlinemedien unter Einschluss des Internet und von Datenbanken/Datenträgern aller Art. Ohne schriftliche Genehmigung des Verlages dürfen diese Publikationen oder Teile daraus nicht in andere Sprachen übersetzt oder in irgendeiner Form mit mechanischen oder elektronischen Mitteln (einschließlich Fotokopie, Tonaufnahme und Mikrofilm) reproduziert oder auf Datenträger oder einem Computersystem gespeichert werden.

Typographische Gestaltung und redaktionelle Bearbeitung werden vom Verlag durchgeführt.

\section{Disclosure Statement}

Die Autoren müssen alle Formen der Unterstützung ihrer Arbeit, inklusive der durch Pharmaunternehmen, angeben; alle Koautoren sollen etwaige Interessenskonflikte offenlegen. Diese Information wird am Ende des Artikels veröffentlicht.

Ethische und rechtliche Voraussetzungen

Manuskripte, bei denen Untersuchungen an Probanden oder Patienten durchgeführ wurden, müssen die Erklärung enthalten, dass das Versuchsprotokoll von einer Ethikkommission genehmigt wurde und den ethischen Standards der Deklaration von Helsinki in ihrer erweiterten Form von 1975 und ihren Zusätzen von 1983, 1989 und 1996 [JAMA 1997:277:925-926] entspricht. Zugleich muss im Manuskripttext auf die Einwilligung de Versuchsperson nach Aufklärung hingewiesen werden. Angaben, die auf die Identität der Person schließen lassen, sind unzulässig.
Im Fall von Tierexperimenten muss von den Autoren angegeben werden, dass diese den jeweils gültigen rechtlichen Standards entsprechen.

\section{Plagiatsrichtlinien}

$\mathrm{Ob}$ absichtlich oder unabsichtlich, der Diebstahl geistigen Eigentums ist ein ernstes Vergehen. Wir definieren einen Artikel, der zu mindestens $25 \%$ mit einer anderen Veröffentlichung übereinstimmt, ohne sie zu zitieren, als Plagiat.

Falls Hinweise auf ein solches Vorgehen vor oder nach der Annahme eines Manuskriptes vorliegen, wird der Autor die Gelegenheit bekommen, dazu Stellung zu nehmen. Wenn seine Argumente nicht zufriedenstellend sind, wird das Manuskript zurückgezogen und dem Autor wird es untersagt, für einen von den zuständigen Editors zu bestimmenden Zeitraum weitere Artikel zu publizieren.

\section{Gliederung}

Titelseite: Die Titelseite muss den Titel und Kurztitel der Arbeit in Deutsch und Englisch enthalten.

Autorennamen und -adressen: Die vollständigen Autorennamen mit der jeweiligen Institutsangabe der Autoren, die vollständige Postadresse des Korrespondenzautors sowie E-Mail-Adresse sollen angegeben sein.

Schlüsselwörter: Zu einer Arbeit gehören 3-8 Schlüsselwörter (Englisch und Deutsch), die möglichst der «Medical Subjects Heading List» des Index Medicus entnommen sein sollen (die Liste ist unter www.nlm.nih.gov erhältlich)

Zusammenfassung: Jedes Manuskript muss eine englische und deutsche Zusammenfassung enthalten, die jeweils 200 Wörter nicht überschreiten. Zusammenfassungen von Original arbeiten sind zu strukturieren in Hintergrund, Patienten und Methoden (oder Material und Methoden), Ergebnisse und Schlussfolgerungen; die von Kasuistiken in Hintergrund, Fallbericht und Schlussfolgerungen.

Fußnoten: Fußnoten sollten nur in Tabellen verwendet werden.

Geschützte Produkt- bzw. Markennamen: Bei ihrem ersten Auftreten im Text müssen geschützte Produkt- und Markennamen mit einem hochgestellten ${ }^{\circledR}$ bzw. ${ }^{\mathrm{TM}}$ bezeichnet werden. Die Verantwortung für die korrekte Befolgung dieser Vorschrift liegt ausschließlich beim Autor.

Abbildungen und Tabellen: Tabellen sind Teil des Textes und sollen am Ende des Manuskriptes eingefügt werden. Abbildungen sollen als separate Dateien eingereicht werden. Die Anzahl der Abbildungen und Tabellen sollte limitiert sein, sie sollen den Text illustrieren. Hinweise auf Abbildungen und Tabellen erfolgen im Text mit (Abb.1) bzw. (Tab. 1). Im Text genannte Informationen bedürfen keiner Wiederholung in einer Tabelle.

Halbtonabbildungen (schwarz-weiß oder farbig) müssen in der endgültigen Größe eine Auflösung von 300 dpi haben, Strichzeichnungen 800-1200 dpi.

Farbabbildungen sind möglich, dem Autor wird jedoch ein Reproduktions- und Druckkostenanteil von EUR 280,- + MWSt. pro Abbildung in Rechnung gestellt.

Literatur

Bei Zitaten im Text werden bis zu zwei Autoren zusammen mit dem Jahr der Publikation in eckigen Klammern erwähnt. Bei drei und mehr Autoren wird nur der Name des Erstautors, gefolgt von «et al.», und der Jahreszahl notiert. Beispiel: [Sauke, 2004; Horn und Mehl, 2004; Jager et al., 2004]

Das Literaturverzeichnis soll nur die im Text zitierten Publikationen enthalten und alphabetisch nach den Namen der Erstautoren geordnet sein. Zitierbar sind nur Arbeiten, die publiziert oder zur Publikation angenommen sind. Der Passus «in Vorbereitung» oder «persönliche Mitteilung» ist zu vermeiden.

Bitte mindestens die ersten drei Autoren jeder Arbeit angeben (Nachname und Initial, ohne Komma oder Punkt), auf weitere Autoren kann mit «et al.» hingewiesen werden.

Die Autoren sind für eine korrekte und vollständige Zitierung der Referenzen verantwortlich.

Die Abkürzung der Zeitschriftentitel erfolgt nach dem Index-Medicus-System (www.nlm.nih.gov).

Beispiele zur Abfassung von Literaturzitaten sind zu finden unter www.karger.com/ ver_guidelines.

Deutsch schreiben - Englisch publizieren

Um die internationale Rezeption ursprünglich deutschsprachiger Arbeiten zu ermöglichen, bietet der Verlag den Autoren an, ihre angenommenen Arbeiten gegen eine Kostenbeteiligung professionell ins Englische übersetzen zu lassen. Die übersetzten Beiträge werden als PDF-Datei kostenlos über die Homepage des Verlages zur Verfügung gestellt. Weitere Informationen erhalten Sie unter www.karger.com/ver

Kosten

Für Artikel, die die maximal vorgesehene Seitenzahl nicht überschreiten, werden keine Druckkosten erhoben (siehe Rubriken und Umfang).

Jede weitere angefangene Druckseite wird dem Autor mit EUR 390,- + MWSt. pro Druckseite in Rechnung gestellt.

\section{Druckfahnen}

Falls nicht anders vereinbart, werden die Druckfahnen dem Korrespondenzautor zugeschickt und sollen schnellstmöglich zurückgesandt werden. Änderungen in den Druckfahnen, die über die Korrektur von Satzfehlern hinausgehen, werden dem Autor in Rechnung gestellt. Es werden keine Umbruchabzüge versandt.

\section{Sonderdrucke}

Ein Formular und die Preisliste zur Sonderdruckbestellung werden mit den Druckfahnen versandt. Bestellungen, die nach Drucklegung bei uns eingehen, können nur zu wesentlich höheren Preisen ausgeführt werden.

\section{KARGER}

Fax +497614520714

Information@Karger.de

www.karger.com

\section{(c) 2012 S. Karger GmbH, Freiburg}

Verfügbar unter:

www.karger.com/ver_guidelines 